\title{
A Case of Multiple Liver Metastases after Surgery in Elderly HER2-Positive Breast Cancer in Which Anastrozole + Trastuzumab Was Ineffective but T-DM1 Was Effective
}

\author{
Tomohiro Shidahara $^{a}$ Shozo Ohsumi ${ }^{a}$ Yuichiro Miyoshi ${ }^{a}$ \\ Mina Takahashi ${ }^{a}$ Seiki Takashima ${ }^{a}$ Kenjiro Aogia Norihiro Teramoto ${ }^{b}$ \\ aDepartment of Breast Oncology, National Hospital Organization Shikoku Cancer Center, \\ Matsuyama, Japan; 'bepartments of Pathology, National Hospital Organization Shikoku \\ Cancer Center, Matsuyama, Japan
}

Keywords

Breast cancer · T-DM1 · Elderly patient $\cdot$ Recurrence

\begin{abstract}
Chemotherapy is often difficult to treat human epidermal growth factor receptor 2 (HER2)positive metastatic recurrent breast cancer in the elderly, and no standard treatment has been established at this point. We experienced a case in which trastuzumab (Tmab) + anastrozole (ANA) was ineffective (progressive disease; PD) in elderly HER2-positive breast cancer with postoperative multiple liver metastases, but T-DM1 was significantly effective (complete response; CR), and treatment could be continued safely. An 82-year-old woman was referred to our department with a right breast mass. A close examination revealed right breast cancer CT1bNOMO cStage I, and total mastectomy and sentinel lymph node biopsy were performed. The postoperative pathological result was pT1bN0M0 pStage I (luminal HER2 type). The patient was elderly and had no adjuvant treatment after the operation. Approximately 2 years after the operation, multiple liver metastases were observed, and treatment with ANA and Tmab was started. Four months later, MRI showed that the number of multiple liver metastases increased. The patient was diagnosed with PD, and the anti-HER2 drug was changed from trastuzumab to trastuzumab emtansine (T-DM1). The dose was reduced due to vomiting (grade 3). Two months later, MRI showed that the multiple liver metastases shrank and became obscure after 5 months. After that, T-DM1 was continued, and the disease did not worsen. In
\end{abstract}


elderly people with difficulty in administering chemotherapy, T-DM1 may have a safe and sufficient therapeutic effect by adjusting the dose and managing side effects appropriately.

(c) 2021 The Author(s).

Published by S. Karger AG, Basel

\section{Introduction}

Chemotherapy is often difficult to administer for treatment of human epidermal growth factor receptor 2 (HER2)-positive metastatic recurrent breast cancer in the elderly, and the standard treatment regimen is not clear. We observed an increase in metastatic lesions after starting Tmab + ANA for multiple liver metastases after surgery for elderly HER2-positive breast cancer, but when we changed the treatment to T-DM1, CR was achieved and was maintained without serious side effects. Therefore, the experience of this case is reported in this study, along with a review of the literature.

\section{Case Report}

\section{Patient History}

The patient was an 82-year-old woman. She noticed a right breast mass and visited a clinic and was referred to our department for consultation in February X. Upon closer examination, she was diagnosed with right breast cancer (cT1bN0M0cStage I). Total right mastectomy + SNL biopsy was performed in March X. As sentinel node biopsy was negative, axillary dissection was omitted. Postoperative pathological results were pT1bN0M0 pStage I (infiltrating ductal carcinoma, NG3, ER >90\%, PR [-], human epidermal growth factor receptor 2 HER2 [3+], and Ki67: 35\%). However, in consideration of age and physical condition, postoperative adjuvant therapy was not performed, and follow-up was performed. Subsequently, the patient progressed without recurrence. However, in February X+ 2 (1 year and 11 months after surgery), PET-CT and MRI showed multiple liver metastases.

Medical history

Medical history included hypertension and chronic renal failure.

Family History

Family history evaluation showed breast cancer (daughter) and stomach cancer (sister).

\section{Image Findings}

Two years after the operation, PET-CT (Fig. 1a, b) and MRI showed multiple liver metastases (Fig. 2a-c), and CA15-3 (8-23 U/mL) tended to increase.

\section{Treatment Policy/Progress}

The patient was diagnosed with multiple liver metastases after surgery for hormone receptor-positive and HER2-positive breast cancer. Because she was old, chemotherapy was not indicated. In March X + 2 (2 years after surgery), anti-HER2 agent and endocrine therapy (Tmab + ANA) were started as the first-line treatment after recurrence. In July X + 2, MRI showed an increase in multiple liver metastases (Fig. 3a, b), which was diagnosed as PD. T-DM1 was selected as the second-line treatment after recurrence. Although there was no 


\section{Case Reports in Oncology}

c

2021 The Author(s). Published by S. Karger AG, Basel www.karger.com/cro

Shidahara et al.: Liver Metastases in Elderly Breast Cancer in Which T-DM1 Was Effective
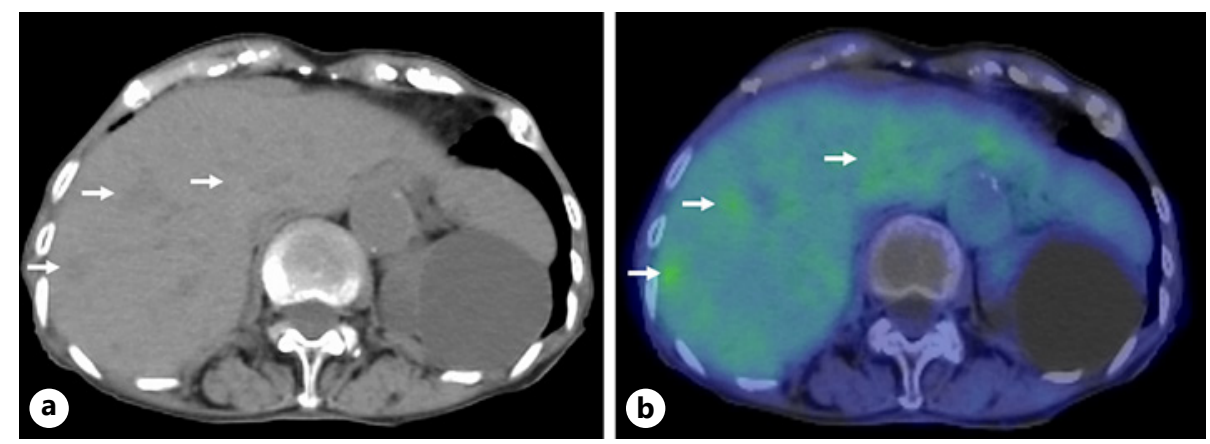

Fig. 1. PET-CT (2 years after surgery): low absorption areas are scattered in the liver (a), accompanied by faint FDG accumulation (b).
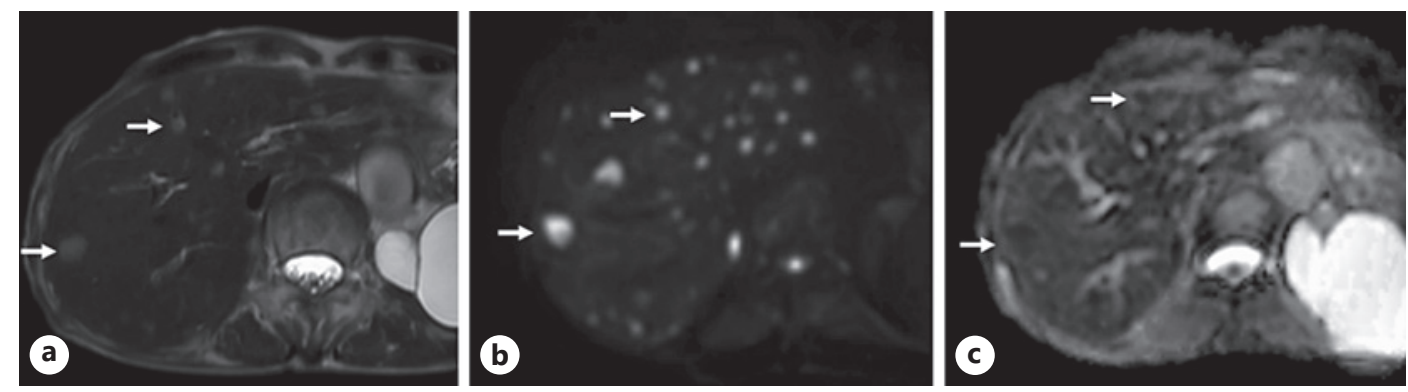

Fig. 2. MR images (2 years after surgery): liver metastasis is shown, and T2WI mild hyperintensity (a), DWI hyperintensity (b), and ADC low (c) nodules are frequently observed in the liver.
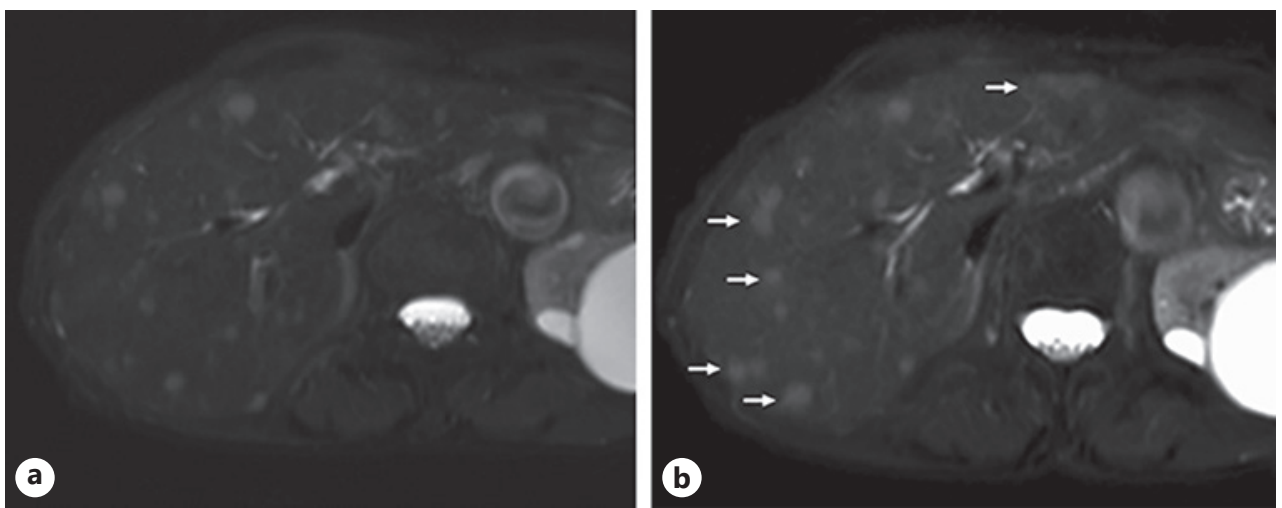

Fig. 3. Progress of the MR image before (a) and after (b) the start of Tmab + ANA: 4 months after the start of Tmab + ANA treatment, more frequent liver metastases are observed compared to before treatment.

history of taxane administration, the administration of T-DM1, which is considered to have relatively fewer side effects, was judged to be appropriate considering age. Tmab + ANA was discontinued in July $X+2$, and administration of T-DM1 $3.6 \mathrm{mg} / \mathrm{kg}$ was started. Because vomiting (grade 3) occurred at the first administration, the dose was reduced to $2.4 \mathrm{mg} / \mathrm{kg}$ at the second administration. Two months after the start of T-DM1 (September X + 2), MRI showed reduction in multiple liver metastases (Fig. 4b) compared with before the start of T-DM1 treatment (Fig. 4a). Three months later (December X + 2), MRI was performed, and 


\section{Case Reports in Oncology}

Shidahara et al.: Liver Metastases in Elderly Breast Cancer in Which T-DM1 Was Effective
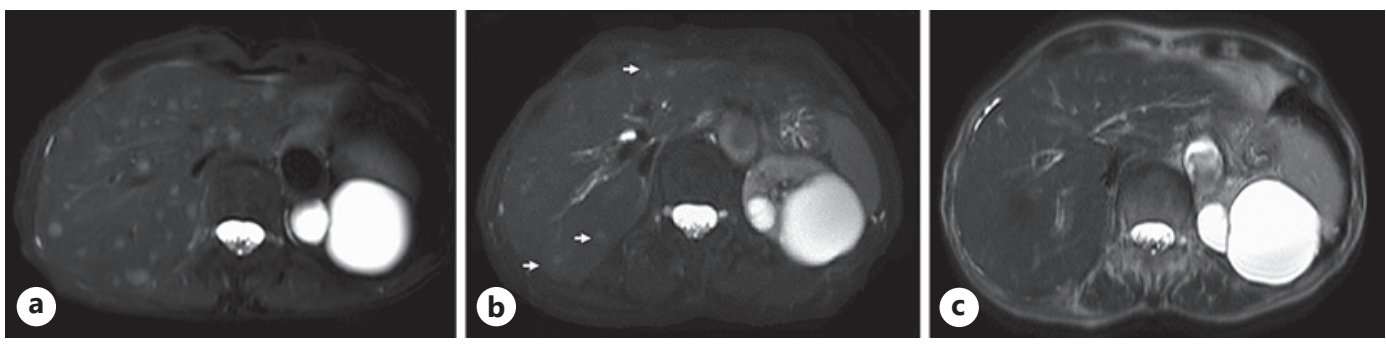

Fig. 4. Progress of MR images before and after the start of T-DM1: compared with before the start of T-DM1 treatment (a), multiple liver metastases shrank 2 months after the start of treatment (b). Five months after the start of treatment, multiple liver metastases are obscure (c) and judged as CR.

the 7 liver metastases were obscure (Fig. 4c), and it was judged as CR. Subsequently, the same dose of T-DM1 (2.4 mg/kg) was continued, and CR was maintained for 10 months or longer without any adverse events.

\section{Discussion}

In T-DM1, which is a drug that can minimize exposure to normal tissues, DM1 (a microtubule polymerization inhibitor) is bound to Tmab. In addition to the effect of Tmab, DM1 is directly taken up by HER2-positive tumor cells, and a stronger anticancer effect is observed. Regarding the use of T-DM1, a double-blind, randomized phase III study of T-DM1 versus capecitabine + lapatinib (control group: XL) in patients with HER2-positive locally advanced or metastatic/recurrent breast cancer $(n=991)$ who had been treated with taxane + trastuzumab (EMILIA study) was conducted. This study showed a significant prolongation of PFS (T-DM1 group 9.6 months and XL group 6.4 months, HR: 0.65; 95\% CI: 0.55-0.77) [1] and OS (T-DM1 group 29.9 months and XL group 25.9 months, HR: 0.75, 95\% CI: 0.64-0.88) [2] in the T-DM1 group and also showed a significant improvement in response rate (T-DM1 group, 43.6\%; XL group, 30.8\%) [1] in the T-DM1 group, and both efficacy and safety were confirmed. Under the current NCCN guidelines, the recommended first-line treatment for HER2-positive metastatic recurrent breast cancer is a combination of anti-HER2 drugs (Tmab, Pmab) + chemotherapy (NCCN category of evidence 1: docetaxel or category 2A: paclitaxel), the second-line treatment is T-DM1, and the third-line treatment is another line of drug therapy + HER-targeted therapy (category 1: tucatinib + Tmab + capecitabine; category 2A: Famtrastuzumab deruxtecan-nxki, neratinib + capecitabine, and Margetuximab-cmkb + chemotherapy) [3].

However, in elderly people, it is often difficult to administer chemotherapy due to mental and physical dysfunction and comorbidities. Endocrine therapy \pm HER2-targeted therapy or other HER2-targeted therapies are recommended by the NCCN guidelines for older patients who are hormone receptor-positive and HER2-positive and are not indicated for chemotherapy [3]. In this case, HER2 therapy (Tmab) + endocrine therapy (AI) was administered in combination based on the guidelines for hormone receptor-positive and HER2-positive breast cancer postoperative multiple liver metastatic disease for which chemotherapy is not indicated due to old age. Multiple liver metastatic lesions increased and became PD. Therefore, Tmab + ANA was discontinued and T-DM1 was started as a second-line treatment, and marked reduction of multiple liver metastases was observed 2 months after the start of T-DM1. Five months after the start of T-DM1, the metastatic lesion was judged to be CR, and T-DM1 administration was continued thereafter without recurrence. Adverse effects caused by T-DM1 
occurred in $87.1 \%$ of patients, including fatigue (41.0\%), nausea (33.7\%), thrombocytopenia (29.6\%), increased AST (20.4\%), and increased ALT (16.1\%) [1]. In this case, vomiting (grade 3) was observed at the first administration of T-DM1, but the adverse effects disappeared after the 2-step dose reduction at the second administration, and the treatment could be continued safely without losing the antitumor effect. However, there is no clear evidence regarding the efficacy or safety of the use of T-DM1 in the elderly. Currently, a comparative study between T-DM1 and pertuzumab + trastuzumab + taxane as the first-line treatment for elderly HER2-positive advanced breast cancer aged $\geq 65$ years [4] is ongoing, and we are awaiting the results on efficacy and safety, and T-DM1 is considered more tolerable in elderly patients with fewer adverse events than chemotherapy because of its mechanism of action. In addition, a phase III study (MARIANNE study $[n=1,095]$ ) comparing T-DM1 with taxane + Tmab was conducted in untreated metastatic breast cancer. In this study, PFS was noninferior to taxane + Tmab even when T-DM1 was used as the first-line treatment (T-DM1 group 14.1 months and control group 13.7 months; T-DM1 group vs. control group, HR: 0.91, 95\% CI: 0.73-1.33). In terms of safety, the toxicity was less in the T-DM1 group than in the control group, with grade 3 or higher (45.4\% vs. 54.1\%), and the period during which QOL could be maintained was significantly longer in the T-DM1 group than in the control group (7.7 vs. 3.6 months, HR: 0.70, 95\% CI: 0.57-0.86) [5].

\section{Conclusion}

T-DM1 was administered to an elderly patient with multiple liver metastases after surgery for HER2-positive breast cancer, in which Tmab + ANA was ineffective. The dose of T-DM1 was reduced due to an adverse effect (vomiting), but CR was achieved and could be maintained without serious adverse effects thereafter. Therefore, even in elderly people with difficulty in administering chemotherapy and who have not been treated with taxane + trastuzumab, T-DM1 may provide a safe and sufficient therapeutic effect by appropriately controlling adverse effects and adjusting the dose.

\section{Acknowledgments}

We would like to express our deep gratitude to the staff of the Department of Breast Oncology and Department of Pathology, Shikoku Cancer Center, for their guidance in writing this manuscript.

\section{Statement of Ethics}

We have reported this case in compliance with the Declaration of Helsinki. Written informed consent was obtained from the patient for publication of this case report and any accompanying images. Case reports are granted an exemption from requiring ethical approval at the National Hospital Organization Shikoku Cancer Center.

\section{Conflict of Interest Statement}

Shozo Ohsumi received lecture fee from AstraZeneca K.K. The authors have no conflicts of interest to declare.

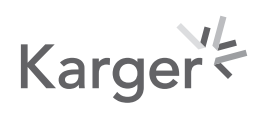




\section{Case Reports in Oncology}

\begin{tabular}{l|l}
\hline Case Rep Oncol 2021;14:1632-1637 \\
\hline DOI: 10.1159/000520357 & $\begin{array}{l}\text { @ 2021 The Author(s). Published by S. Karger AG, Basel } \\
\text { www.karger.com/cro }\end{array}$ \\
\hline
\end{tabular}

Shidahara et al.: Liver Metastases in Elderly Breast Cancer in Which T-DM1 Was Effective

Funding Sources

The authors declare no funding sources to report.

\section{Author Contributions}

T.S. wrote the main text of the manuscript and collected photographs. T.S. and S.O. did the final revision of the text. All authors approved the final version of the manuscript.

\section{Data Availability Statement}

All data relevant to the case presentation are available as part of the article, and no additional source data are required.

\section{References}

1 EMILIA Study Group; Verma S, Miles D, Gianni L, Krop IE, Welslau M, Baselga J, et al. Trastuzumab emtansine for HER2-positive advanced breast cancer. N Engl J Med. 2012;367(19):1783-91.

2 Diéras V, Miles D, Verma S, Pegram M, Welslau M, Baselga J, et al. Trastuzumab emtansine versus capecitabine plus lapatinib in patients with previously treated HER2-positive advanced breast cancer (EMILIA): a descriptive analysis of final overall survival results from a randomised, open-label, phase 3 trial. Lancet Oncol. 2017;18:732-42.

3 National Comprehensive Cancer Network. NCCN clinical practice guidelines in oncologyTM breast cancer version 5. National Comprehensive Cancer Network; 2021. https://www.nccn.org/professionals/physician_ gls/pdf/breast.pdf Accessed 2021 Aug 12.

4 Shimomura A, Tamura K, Mizutani T, Shibata T, Hara F, Fujisawa T, et al. A phase III study comparing trastuzumab emtansine with trastuzumab, pertuzumab, and docetaxel in elderly patients with advanced stage HER2-positive breast cancer: (JCOG1607 HERB TEA study). Jco. 2019;37(15_Suppl):TPS1100.

5 Perez EA, Barrios C, Eiermann W, Toi M, Im YH, Conte P, et al. Trastuzumab emtansine with or without pertuzumab versus trastuzumab plus taxane for human epidermal growth factor receptor 2-positive, advanced breast cancer: primary results from the phase III MARIANNE study. J Clin Oncol. 2017;35:141-8. 